\title{
GASTRODUODENAL TOLERABILITY OF NIMESULIDE AND DICLOFENAC IN PATIENTS WITH OSTEOARTHRITIS
}

\author{
ARMANDO PORTO, ${ }^{1}$ CONCEIÇÃO REIS, ${ }^{1}$ RUI PERDIGOTO,${ }^{1}$ MORNA GONÇALVES, ${ }^{2}$ \\ PEDRO FREITAS, ${ }^{3}$ AND ALBERTO MACCIOCCHI ${ }^{4}$ \\ ${ }^{1}$ Coimbra University Hospitals, Coimbra, ${ }^{2}$ Leiria District Hospital, Leiria, ${ }^{3}$ Medical \\ Department, Rhône-Poulenc Rorer, Lisbon, Portugal, and ${ }^{4}$ Product Development, Helsinn \\ Healthcare SA, Pazzallo, Switzerland
}

\begin{abstract}
This 1-month, randomized, double-masked, parallel-group study was conducted to compare nimesulide ( $100 \mathrm{mg}$ twice daily) with diclofenac ( $50 \mathrm{mg}$ three times daily) with respect to gastroduodenal tolerability and efficacy in patients with osteoarthritis. Results of gastroduodenal endoscopy in 83 patients (42 receiving nimesulide, 41 receiving diclofenac) revealed that, after 30 days, 4 patients (1 nimesulide, 3 diclofenac) had developed ulcers and 6 patients (4 nimesulide, 2 diclofenac) had developed erosions; however, differences between the treatment groups were not statistically significant. Both study drugs were well tolerated. Ten patients (5 in each group) withdrew from the study prematurely because of adverse events. Efficacy was assessed by measuring pain on visual analogue scales, using the functional index of Lequesne, and by scoring spontaneous pain, pain on passive movement, and functional impairment. Nocturnal pain was also checked. All efficacy variables showed a significant improvement during the study, and no statistically significant differences were observed between the treatment groups. Key words: nimesulide, diclofenac, gastroduodenal tolerability, osteoarthritis.
\end{abstract}

\section{INTRODUCTION}

Nonsteroidal anti-inflammatory drugs (NSAIDs) inhibit prostaglandin (PG) synthesis by blocking cyclooxygenase (COX) pathways. Although beneficial in reducing pain and inflammation in arthritic joints, decreased PG formation has potentially scrious consequences in the gastrointestinal mucosa, where endogenous PGs (mainly prostaglandin $\mathrm{E}_{2}\left[\mathrm{PGE}_{2}\right]$, produced by COX-1) are involved in maintaining protective mechanisms. ${ }^{1-3} \mathrm{PGE}_{2}$ promotes the production of mucus and bicarbonate and, together with prostaglandin $\mathrm{I}_{2}\left(\mathrm{PGI}_{2}\right)$ helps to maintain mucosal vasodilation; $\mathrm{PGE}_{2}$

Address correspondence to: A. Macciocchi, MD MFPM, Products Development, Helsinn Healthcare SA, Via Pian Scairolo, P.O. Box 357, 6915 Pambio-Noranco, Switzerland. Received for publication on March 13, 1998. Printed in the USA. Reproduction in whole or part is not permitted. 
alone may play a role in reducing acid secretion. Therefore, an NSAID that exerts a weaker effect on COX-1 than on COX-2 (inflammatory-PG producing) activity might reduce the risk of lesions in the gastrointestinal tract.

Nimesulide is an NSAID belonging to a new chemical class. It is weakly acidic ( $\mathrm{pKa}=6.5$ ) because of its sulfonanilide functional group. In rats, at pharmacologically active doses, nimesulide does not alter the amounts of $\mathrm{PGE}_{2}, \mathrm{PGI}_{2}$, or thromboxane $\mathrm{A}_{2}$ in the gastric mucosa. ${ }^{4}$ In comparison with other NSAIDs, nimesulide demonstrates little ulcerogenic activity in rats. ${ }^{5,6}$

Data on COX-2/COX-1 inhibition show preferential activity of nimesulide on COX-2: the COX-2/COX-1 ratio varies according to the type of model from 0.7 to $0.0004 .^{7,8} \mathrm{COX}-2$ selectivity of nimesulide was confirmed recently in a controlled study in humans. ${ }^{9}$

Endoscopic studies in humans confirmed the tolerability of nimesulide in the gastrointestinal tract. In a double-masked, comparative trial ${ }^{10}$ using high dosages of nimesulide (up to $400 \mathrm{mg} / \mathrm{d}$ ) or placebo administered for 1 week to dyspeptic patients, no between-group difference in gastric mucosal damage was observed gastroscopically. A single-masked study ${ }^{11}$ concluded that nimesulide $200 \mathrm{mg} / \mathrm{d}$ was as effective as indomethacin 150 $\mathrm{mg} / \mathrm{d}$ in the treatment of articular inflammatory disease but caused less gastric mucosal damage.

The primary aim of the present study was to assess the gastroduodenal tolerability of nimesulide in patients with osteoarthritis (OA). A secondary aim was to compare the efficacy of nimesulide with that of diclofenac.

\section{PATIENTS AND METHODS}

This study was designed to be a multicenter, randomized, double-masked comparison of parallel groups that included approximately 100 patients with OA of the hip or knee. The patients were separated into blocks of 10 and randomly assigned to receive either nimesulide $100 \mathrm{mg}$ twice daily (BID) or diclofenac $50 \mathrm{mg}$ three times daily (TID) according to a computergenerated code. The study medications were visually indistinguishable and had identical packaging and labeling. Each participating patient received two boxes of medication marked box $A$, which contained 70 tablets (35 for morning and 35 for evening administrations), and one marked box $\mathrm{B}$, which contained 35 tablets for administration at midday. Patients in the nimesulide group received a visually identical placebo tablet to take at midday. Enough medication was supplied for an additional week after the study period. If required, patients were permitted to take paracetamol 500 $\mathrm{mg} / \mathrm{tablet}$, to a maximum of 6 tablets/d, for additional analgesia. 
Patients of either sex, $\geqslant 50$ years of age, were recruited for the study if they had given their written informed consent to participate and had undergone a washout period following their previous NSAID therapy (48 hours for nonoxicam-class NSAIDs and 72 hours for oxicam-class NSAIDs). Patients also had to fulfill the following entry criteria: endoscopic findings of normal gastroduodenal mucosa or $\leqslant 10$ petechiae (scored 0 or 1; Table I); OA of the hip or knee, with painful exacerbation for more than 1 week or requirement for treatment for 1 month with an NSAID, and with daily pain (spontaneous or on movement) and functional impairment of the affected joint; spontaneous pain intensity scored $\geqslant 4.9 \mathrm{~cm}$ on a $10-\mathrm{cm}$ Huskisson-type ${ }^{12}$ visual analogue scale (VAS); and lesions rated 1 to 3 (Table II) seen on radiologic examination for $\leqslant 1$ year before study entry.

Patients were excluded from the study if their OA had been present for more than 1 year, if they had severe or incapacitating OA (unable to walk) necessitating surgical intervention during the study period, or if they were being treated with intra-articular corticoids during the 4 weeks before study entry. Patients with severe hepatic, renal, cardiovascular, endocrine, or hematologic diseases or bronchial asthma were excluded from the study, as were patients with a known history of hypersensitivity reactions to NSAIDs (particularly to aspirin or derivatives of propionic acid). Pregnant women, nursing mothers, or women who might become pregnant were also excluded.

The use of anticoagulants, hydantoin, oral antidiabetics, antimalarials, other NSAIDs, immunosuppressive agents, central or peripheral analgesics, systemic or intra-articular corticoids, muscle relaxants, neuroleptics, or antidepressants was prohibited during the study. All other medications were permitted. Patients were allowed to continue physiotherapeutic measures initiated at least 1 month before study entry.

Participants were scheduled for three visits:

1. Day -7: Patients underwent endoscopy to determine the status of the gastroduodenal mucosa.

Table I. Scoring criteria for gastroduodenal mucosa as assessed endoscopically.

\begin{tabular}{lc}
\hline \hline \multicolumn{1}{c}{ Criteria } & Grade \\
\hline Normal mucosa & 0 \\
$1-10$ Petechiae & 1 \\
$>10$ Petechiae & 2 \\
$1-5$ Erosions & 3 \\
$6-10$ Erosions & 4 \\
$11-25$ Erosions & 5 \\
D25 Erosions & 6 \\
Uicer & 7 \\
\hline \hline
\end{tabular}


Table II. Scoring criteria for assessment of osteoarthritis of the knee or hip as assessed radiologically.

\begin{tabular}{|c|c|}
\hline Criteria & Grade \\
\hline Normal & 0 \\
\hline Slight narrowing of joint space, very slight osteophytosis & 1 \\
\hline Clear narrowing of joint space, evident osteophytosis, slight osteosclerosis & 2 \\
\hline \multirow{2}{*}{\multicolumn{2}{|c|}{ Marked narrowing of joint space, evident osteophytosis, periarticular osteosclerosis }} \\
\hline & 3 \\
\hline \multicolumn{2}{|l|}{ Serious narrowing of joint space, severe osteophytosis, marked periarticular } \\
\hline osteosclerosis with geodes, severe deformation of bone extremities & 4 \\
\hline
\end{tabular}

2. Day 0 (baseline): Routine hematologic and serum biochemical analyses and urinalysis were performed, and patients began taking their study medication.

3. Day 30 (final): On completion of the study, patients returned any unused medication (to establish compliance), underwent repeat gastroduodenal endoscopy and laboratory testing, and answered nonleading questions about adverse events (AEs), which were graded according to their severity, frequency, relationship to the study medication, and outcome.

Both endoscopic procedures (day -7 and day 30 ) were performed by the same investigator to reduce scoring bias. The investigator also assessed the tolerability of the treatment according to a 4-point verbal scale (excellent, good, fair, poor).

Compliance was assessed as good if the patient returned $\leqslant 6$ tablets/ mo, fair if 7 to 12 tablets, and poor if $>12$ tablets (excluding the 21 supplementary tablets provided for an additional week of treatment).

\section{Efficacy Variables}

Spontaneous pain was assessed using a $10-\mathrm{cm}$ VAS. Pain on passive motion and functional impairment were assessed using a 5-point verbal scale (absent, mild, moderate, severe, very severe). Functional status was assessed using the functional index of Lequesne for OA of the hip and knee (25-point scale: $0=$ healthy subjects, $24=$ worst possible case).$^{13}$ The presence of nocturnal pain was checked, and the number of awakenings due to pain was recorded. The administration of paracetamol for analgesia was analyzed as a secondary efficacy variable.

\section{Statistical Analysis}

Data entry was done using the dBase $\mathrm{IV}^{\circledR}$ software program (Ashton Tate Corp., Maidenhead, United Kingdom), and the statistical analysis was performed using SOLO 4.0 (BMDP ${ }^{\circledR}$ Statistical Software Inc., Los Angeles, California) and Testimate ${ }^{\circledR}$ (Institut fur Datenanalyse und 
Versuchsplanung [IDV], Gauting, Germany) on an IBM PS2 personal computer.

The variables assessed for the two treatment groups were compared using several statistical tests. Age, body weight, and height were assessed using Student's $t$ test for unpaired data, whereas demographic characteristics such as sex and diagnosis were assessed using the chi-square test. For the safety variables, the Mann-Whitney $U$ test was used for endoscopic scores and judgment; laboratory data were assessed using Student's $t$ test for paired data. For three measures of efficacy-VAS, functional index, and pain scores-within-group differences were assessed using Wilcoxon's rank sum test and between-group differences were assessed using the Mann-Whitney $U$ test; judgment and compliance data were assessed using the Mann-Whitney $U$ test.

All tests were two-tailed, and a significance level of 0.05 was chosen.

\section{RESULTS}

Eighty-nine patients ( 14 men and 75 women) entered the study: 44 were randomized to receive nimesulide and 45 to receive diclofenac. However, 1 patient in the diclofenac group was excluded from both the safety and efficacy analyses because of changed dosage, which was considered a major protocol violation. Safety analyses were performed on 88 patients, although final endoscopic findings were available for only 83 patients. Efficacy analyses were performed on data from 77 patients. Six patients with endoscopic findings were treated only 10 to 15 days; therefore, they were included in the safety analysis but not in the efficacy analysis. The two treatment groups were similar with respect to demographic characteristics and localization of OA. Thirty-six patients $(82 \%)$ in the nimesulide group and 35 patients $(78 \%)$ in the diclofenac group had OA of the knee. In patients with bilateral involvement of the hip or knee, the more affected joint was assessed.

\section{Safety Profile}

\section{Clinical Tolerability}

Twenty-two patients (11 in each group) experienced one or more AEs, which generally affected the gastrointestinal tract; 5 of the 11 patients in each group discontinued treatment prematurely because they developed ulcers ( 1 nimesulide, 3 diclofenac) or erosions ( 4 nimesulide, 2 diclofenac). Table III summarizes the incidence of AEs and their severity. The relationship of AEs to the study medication as judged by the investigator is shown in Table IV. 
Table III. Incidence and severity of adverse events (AEs) reported during the study.

\begin{tabular}{lcc}
\hline \hline \multicolumn{1}{c}{ Adverse Events } & $\begin{array}{c}\text { Nimesulide } \\
(\mathbf{n}=\mathbf{4 4})\end{array}$ & $\begin{array}{c}\text { Dicioienac } \\
(\mathbf{n}=\mathbf{4 4})^{*}\end{array}$ \\
\hline Incidence & & \\
Gastralgia & 5 & 7 \\
Nausea & 0 & 2 \\
Sensation of fullness/bloating & 2 & 3 \\
Abdominal pain & 2 & 1 \\
Dizziness & 3 & 1 \\
Headaches & 2 & 1 \\
Skin disorders & 2 & 0 \\
Cardiovascular disorders & 3 & 3 \\
Others & 21 & 19 \\
Total AEs & 21 & 7 \\
Severity & & 8 \\
Mild & 3 & 4 \\
Moderate & 11 & 7 \\
Severe & 7 & 4 \\
\hline \hline
\end{tabular}

* One patient was withdrawn from the study because of a protocol violation.

\section{Gastroduodenal Tolerability}

Patients were included in the study only if the results of the baseline endoscopic examination (day -7) were normal (Table V). At day 30, most of the patients showed normal endoscopic findings, although 4 patients ( 1 nimesulide, 3 diclofenac) had developed ulcers. Erosions were observed in 6 patients ( 4 nimesulide, 2 diclofenac). However, the between-group differences in endoscopic findings were not statistically significant.

\section{Biologic Tolerability}

Statistical analyses were not undertaken because the laboratory tests were performed at different facilities, using different normal ranges. Hematologic variables were normal at baseline and remained within the normal range following treatment in all patients except two (one in each group) who had increased erythrocyte sedimentation rates on day 30 . Serum aminotransferases were normal at baseline for all patients. However, by day 30 , one of the diclofenac-treated patients showed a moderate increase and one of the nimesulide-treated patients showed a marked in-

Table IV. Relationship of adverse events to the study medication, as judged by the investigators.

\begin{tabular}{cccccc}
\hline \hline Drug & Definite & Probable & Doubtful & $\begin{array}{c}\text { Not } \\
\text { Related }\end{array}$ & Total \\
\hline Nimesulide & 12 & 6 & 3 & 0 & 21 \\
Diclofenac & 12 & 5 & 1 & 1 & 19 \\
\hline \hline
\end{tabular}


Table V. Scoring of gastroduodenal mucosa as assessed by endoscopy.

\begin{tabular}{|c|c|c|c|c|}
\hline \multirow[b]{2}{*}{ Grade* } & \multicolumn{2}{|c|}{ Nimesulide } & \multicolumn{2}{|c|}{ Diclofenac } \\
\hline & $\begin{array}{c}\text { Day 0 } \\
\text { (Baseline) }\end{array}$ & $\begin{array}{l}\text { Day 30 } \\
\text { (Final) }\end{array}$ & $\begin{array}{c}\text { Day 0 } \\
\text { (Baseline) }\end{array}$ & $\begin{array}{l}\text { Day } 30 \\
\text { (Final) }\end{array}$ \\
\hline $\begin{array}{l}0 \\
1\end{array}$ & $\begin{array}{c}43 \\
1\end{array}$ & $\begin{array}{c}36 \\
1\end{array}$ & $\begin{array}{r}44 \\
-\end{array}$ & 36 \\
\hline 2 & - & - & - & - \\
\hline 3 & - & 3 & - & 1 \\
\hline 4 & - & 1 & - & 1 \\
\hline 5 & - & - & - & - \\
\hline 6 & - & - & - & - \\
\hline 7 & - & 1 & - & 3 \\
\hline Total & 44 & 42 & $44 \dagger$ & 41 \\
\hline
\end{tabular}

crease in these levels; the latter patient showed no other abnormalities while the diclofenac-treated patient showed an increase in serum aminotransferase, alkaline phosphatase, gamma-glutamyltransferase $(\gamma \mathrm{GT})$, and creatinine levels. Three patients in the diclofenac group showed an increase in serum alkaline phosphatase compared with baseline levels.

Three patients in the nimesulide group and one in the diclofenac group showed increased levels of $\gamma$ GT at day 0 that remained high at day 30 . Two other patients in the nimesulide group had elevated baseline $\gamma$ GT levels that normalized by the end of treatment, whereas two patients in the diclofenac group had normal $\gamma$ GT levels at baseline but elevated levels by the end of the study.

Serum bilirubin levels for all patients remained within the normal range during the study period. One nimesulide-treated patient had an unexplained increase in serum glucose level at day 30 , although she had withdrawn from the study at day 10 because of a severe AE. In the diclofenac group, 1 patient had increased serum cholesterol and creatinine levels, 1 patient had an increased serum cholesterol level, and 1 patient had a slight increase in serum uric acid level at day 30 .

Urinalysis revealed slight proteinuria at baseline in five nimesulidetreated patients and six diclofenac-treated patients. The proteinuria worsened in one patient after receiving diclofenac therapy.

\section{Judgment of Tolerability}

At the end of the study, the investigator expressed his or her opinion regarding the clinical and biologic safety profile of the study medication. No statistically significant differences between the two treatments were identified. The assessment of biological tolerability substantially reflected 


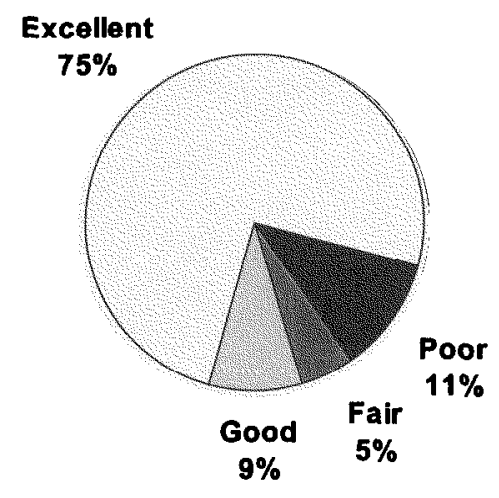

Nimesulide $(n=44)$

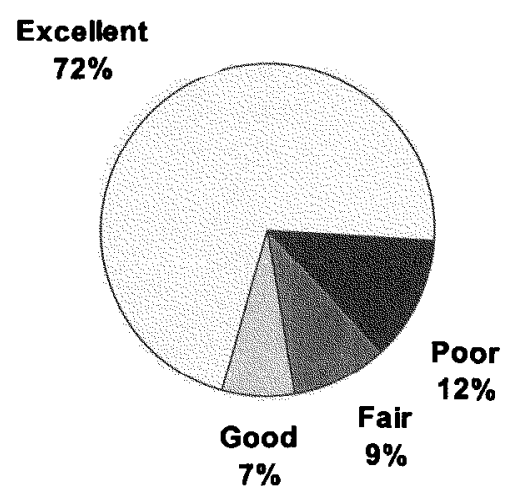

Diclofenac $(n=43)$

Figure 1. Physicians' assessment of clinical tolerability among patients with normal baseline endoscopic findings.

the clinical assessment (excellent in $95.1 \%$ in the nimesulide group and $90 \%$ in the diclofenac group) (Figure 1).

\section{Efficacy}

Based on patient self-assessment using the VAS, spontaneous pain decreased significantly in both groups (nimesulide, $P<0.01$; diclofenac, $P<0.1$ ) following 4 weeks of treatment (Figure 2). The level of spontaneous pain did not differ significantly between the two groups. Similarly, the functional index total score decreased significantly in both groups (nimesulide, $P<0.01$; diclofenac, $P<0.1$ ), but the difference in the treatment effect of the two drugs was not statistically significant. All other measurements of efficacy showed a significant improvement following 4 weeks of treatment (nimesulide, $P<0.01$; diclofenac, $P<0.1$ ), but no statistically significant differences in efficacy were observed between the two groups (Table VI).

At day 30, both the investigator and the patient expressed their judgment regarding the efficacy of the treatment (Table VII). According to the investigators, positive (excellent or good) outcomes were observed in 28 $(71.8 \%)$ of the patients treated with nimesulide compared with $28(73.7 \%)$ of those treated with diclofenac. Similar opinions were expressed by the patients: $28(71.8 \%)$ of the nimesulide-treated patients and $27(71.1 \%)$ of the diclofenac-treated patients recorded a positive outcome. No statistically significant between-group differences in efficacy were found. Five patients ( 3 nimesulide, 5 diclofenac) needed additional analgesia (paracetamol); no statistical assessment was performed because of the small number of patients involved.

Among the nimesulide-treated patients, $30(76.9 \%)$ were assessed as 


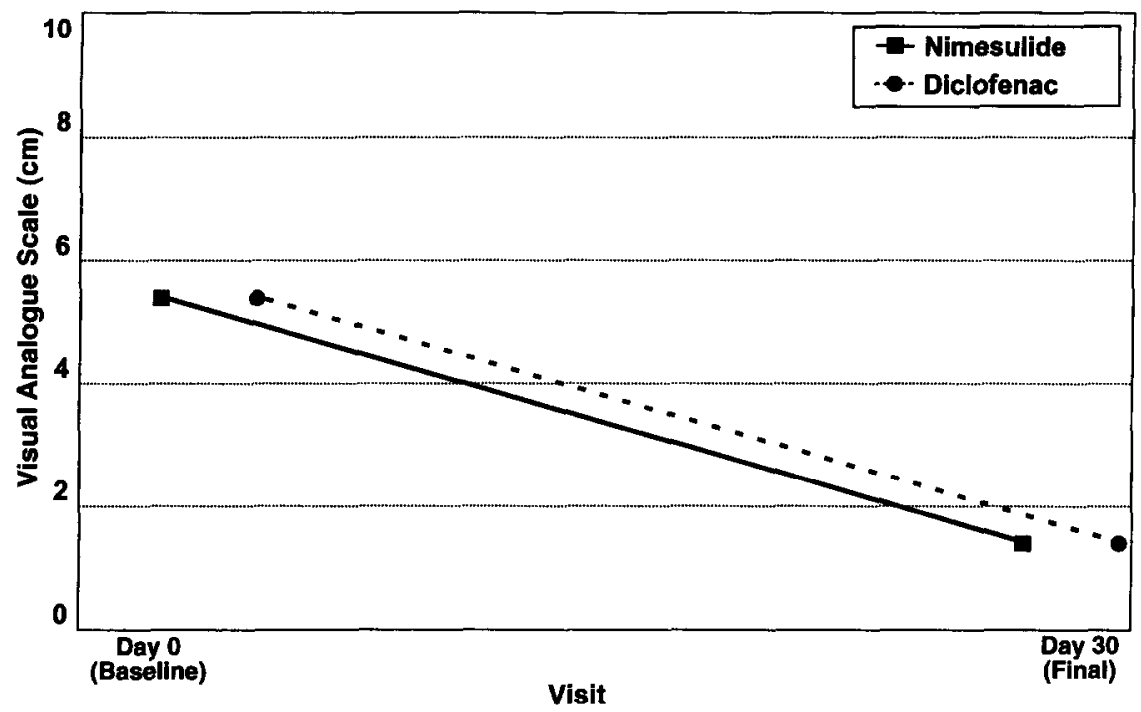

Figure 2. Assessment (mean) of spontaneous pain as recorded by the patients using the visual analogue scale.

compliant with the medication regimen; one patient (2.6\%) was classified as poorly compliant. Among the diclofenac-treated patients, 29 (76.3\%) were classified as compliant, whereas no patient in this group was considered poorly compliant. No significant between-group differences with respect to compliance were found.

Table VI. Measurements of efficacy as reflected in mean (median) pain scores by treatment group at baseline (day 0 ) and study completion (day 30 ).

\begin{tabular}{|c|c|c|c|c|}
\hline & \multicolumn{2}{|c|}{ Nimesulide $(\mathrm{n}=39$ ) } & \multicolumn{2}{|c|}{ Diclofenac $(n=38$ ) } \\
\hline & $\begin{array}{c}\text { Day 0 } \\
\text { (Baseline) }\end{array}$ & $\begin{array}{l}\text { Day 30* } \\
\text { (Final) }\end{array}$ & $\begin{array}{c}\text { Day 0 } \\
\text { (Baseline) }\end{array}$ & $\begin{array}{l}\text { Day 30† } \\
\text { (Final) }\end{array}$ \\
\hline $\begin{array}{l}\text { Lequesne functional index }{ }^{13} \ddagger \\
\text { Spontaneous pain§ } \\
\text { Pain on passive movement" } \\
\text { Pain during the night }\end{array}$ & $\begin{array}{l}16.0(15.5) \\
4.33(4) \\
3.94(4)\end{array}$ & $\begin{array}{l}9.4(10.0) \\
2.74(3) \\
2.15(2)\end{array}$ & $\begin{array}{l}15.1(15.5) \\
4.18(4) \\
3.84(4)\end{array}$ & $\begin{array}{l}8.9(8.25) \\
2.63(3) \\
2.13(2)\end{array}$ \\
\hline $\begin{array}{l}\text { Yes } \\
\text { No } \\
\text { No. of awakenings due to pain }\end{array}$ & $\begin{array}{l}36 \\
3 \\
3.51\end{array}$ & $\begin{array}{l}16 \\
23 \\
0.58\end{array}$ & $\begin{array}{l}36 \\
2 \\
3.07\end{array}$ & $\begin{array}{l}15 \\
23 \\
0.63\end{array}$ \\
\hline
\end{tabular}

Note: Six patients included in the safety analysis were not included in the efficacy analysis because the treatment duration lasted 10 to 15 days.

\footnotetext{
* $P<0.01$

$+P<0.1$.

25-point scale: 0 = healthy subjects, 24 = worst possible case.

$\$$ Assessed based on a $10-\mathrm{cm}$ visual analogue scale.

H 1 =absent; 2 = mild; 3 = moderate; 4 = severe; 5 = very severe.
} 
Table VII. Final assessment of efficacy as judged by the investigators and the patients.

\begin{tabular}{|c|c|c|c|c|c|}
\hline & Excellent & Good & Moderate & Inefiective & $P^{*}$ \\
\hline \multicolumn{6}{|l|}{ Investigators } \\
\hline $\begin{array}{l}\text { Nimesulide }(n=39) \\
\text { Diclofenac }(n=38)\end{array}$ & $\begin{array}{l}13 \\
19\end{array}$ & $\begin{array}{r}15 \\
9\end{array}$ & $\begin{array}{r}10 \\
7\end{array}$ & $\begin{array}{l}1 \\
3\end{array}$ & 0.7304 \\
\hline $\begin{array}{l}\text { Patients } \\
\text { Plimaling }\end{array}$ & & & & & \\
\hline $\begin{array}{l}\text { Nimesulide }(n=39) \\
\text { Diclofenac }(n=38)\end{array}$ & $\begin{array}{l}17 \\
19\end{array}$ & $\begin{array}{r}11 \\
8\end{array}$ & $\begin{array}{r}11 \\
7\end{array}$ & $\begin{array}{l}0 \\
4\end{array}$ & 0.442 \\
\hline
\end{tabular}

Note: Six patients included in the safety analysis were not included in the efficacy analysis because the treatment duration lasted 10 to 15 days.

$* P$ value assessed by Mann-Whitney $U$ test.

\section{DISCUSSION AND CONCLUSIONS}

The present study shows that nimesulide (100 mg BID) for 1 month is as efficacious as diclofenac (50 $\mathrm{mg}$ TID) in relieving the painful symptoms of $\mathrm{OA}$ and may have slightly better gastroduodenal tolerability than diclofenac.

Nimesulide has been demonstrated in animals and humans to have low ulcerogenic potential. The aim of the present study was to compare nimesulide with diclofenac with respect to gastroduodenal tolerability and efficacy in the treatment of OA. The 1-month study period was sufficient for ulcerogenic lesions to develop, although the small number of patients made it impossible to demonstrate a statistically significant difference in gastroduodenal tolerability between the two drugs. The study results could, however, be interpreted as nimesulide having a slightly lower ulcerogenic potential than diclofenac. The same number of patients in each treatment group experienced a worsening in their gastroduodenal mucosa, but a smaller percentage of nimesulide-treated patients (2.3\%) than diclofenac-treated patients $(6.8 \%)$ developed ulcers. Furthermore, the incidence of gastrointestinal-related AEs was lower with nimesulide (42.8\%) than with diclofenac $(68.4 \%)$. It is difficult to determine whether these differences are correlated with the COX-2 selectivity of nimesulide, because diclofenac is an almost indifferent inhibitor of both COX-1 and COX-2. A comparison with a more selective COX-1 inhibitor, also investigating the effects on gastric and systemic PGs, might provide a definitive answer.

Assessment of biologic tolerability revealed a slightly higher but statistically insignificant incidence of abnormalities in the diclofenac group than the nimesulide group. The efficacy of the two drugs was similar. A significant improvement at day 30 compared with baseline was apparent for all efficacy variables, but no statistically significant difference was observed between the two treatments. Previous double-masked studies $^{14,15}$ of the efficacy of nimesulide in the treatment of OA have demonstrated efficacy comparable to that of etodolac, piroxicam, and ketoprofen. 
The use of NSAIDs has expanded dramatically in the past decade and has been accompanied by a proliferation in the number of therapeutic alternatives. However, a significant proportion of patients developed AEs, primarily in the upper gastrointestinal tract, ranging from intolerance to life-threatening perforations and bleeding. NSAID-induced gastropathy is often asymptomatic, with little correlation between dyspeptic symptoms and endoscopic lesions. Studies ${ }^{16-22}$ indicate that at least $22 \%$ of patients taking NSAIDs chronically will develop mucosal erosions, peptic ulcerations, perforations, or bleeding. However, the duration of NSAID therapy is not a dependable marker for the risk of gastropathy, because $25 \%$ of patients with a bleeding peptic ulcer had received NSAID therapy for less than 1 month.

Because of our current experience with NSAIDs, any new drug that has a demonstrably reduced potential for inducing gastropathy and is shown to be as efficacious as those currently prescribed will have a ready, worldwide market.

\section{Acknowledgment}

The study was supported by a research grant from Rhône-Poulenc Rorer, Lisbon, Portugal.

\section{References:}

1. Whittle BJR, Vane JR. A biochemical basis for the gastrointestinal toxicity of nonsteroidal antirheumatoid drugs. Arch Toxicol Suppl. 1984;7:315-322.

2. Smith WL, Meade EA, DeWitt DL. Interactions of PGH synthase isozymes-1 and -2 with NSAIDs. Ann NY Acad Sci. 1994;744:50-57.

3. Isakson PC. Synthesis and pharmacology of selective COX-2 inhibitors. Med Chem Res. 1996;5:344-350.

4. Swingle KF, Moore GGI. Pre-clinical pharmacological studies with nimesulide. Drugs Exp Clin Res. 1984;10:587-597.

5. Böttcher I, Schweizer A, Glatt M, Verner H. A sulphonamido-indanone derivative CGP 28237 (ZK 34228), a novel nonsteroidal anti-inflammatory agent without gastrointestinal ulcerogenicity in rats. Drugs Exp Clin Res. 1987;13:237-245.

6. Rainsford $\mathrm{KD}$. The comparative gastric ulcerogenic activities of non-steroidal antiinflammatory drugs. Agents Actions. 1977;7:573-577.

7. Huff R, Collins P, Kramer S, et al. A structural feature of N[2-(cyclohexyloxy)-4nitrophenyl] methanesulfonamide (NS-398) that governs its selectivity and affinity for cyclooxygenase 2 (COX2). Inflamm Res. 1995;44(Suppl 2):145-146.

8. Grossman CJ, Wiseman J, Lucas FS, et al. Inhibition of constitutive and inducible cyclooxygenase activity in human platelets and mononuclear cells by NSAIDs and COX 2 inhibitors. Inflamm Res. 1995;44:253-257.

9. Cullen I, Kelly L, Coyle D, et al. Selective suppression of cyclooxygenase-2 during chronic 
administration of nimesulide in man. Presented at 4th International Congress on Essential Fatty Acids and Eicosanoids; July 20-24, 1997; Edinburgh, Scotland.

10. Marini U, Spotti D. Gastric tolerability of nimesulide. A double-blind comparison of 2 oral dosage regimens and placebo. Drugs. 1993;46(Suppl 1):249-252.

11. Cipollini F, Mecozzi V, Altilia F. Endoscopic assessment of the effects of nimesulide on the gastric mucosa: Comparison with indomethacin. Curr Ther Res. 1989;45:1042-1048.

12. Huskisson EC. Measurement of pain. Lancet. 1974;2:1127-1131.

13. Lequesne M, Méry C, Samson M, Gérard P. Indexes of severity for osteoarthritis of the hip and knee. Validation-value in comparison with other assessment tests. Scand $J$ Rheumatol. 1987;65(Suppl):85-89.

14. Dreiser RL, Riebenfeld D. Nimesulide in the treatment of osteoarthritis. Double-blind studies in comparison with piroxicam, ketoprofen and placebo. Drugs. 1993;46(Suppl 1): 191-195.

15. Lücker PW, Pawlowski C, Friederich I, et al. Double-blind, randomized, multicentre clinical study evaluating the efficacy and tolerability of nimesulide in comparison with etodolac in patients suffering from osteoarthritis of the knee. Eur J Rheumatol Inflamm. 1994;14:29-38.

16. Bartle WR, Gupta AK, Lazor J. Nonsteroidal anti-inflammatory drugs and gastrointestinal bleeding. A case-control study. Arch Intern Med. 1986;146:2365-2367.

17. Caruso I, Porro GB. Gastroscopic evaluation of anti-inflammatory agents. BMJ. 1980; 280:75-77.

18. Coles LS, Fries JF, Kraines RG, et al. From experiment to experience: Side effects of nonsteroidal anti-inflammatory drugs. Am J Med. 1983;74:820-828.

19. Graham DY, Agrawal NM, Roth SH. Prevention of NSAID-induced gastric ulcer with misoprostol: Multicentre, double-blind, placebo-controlled trial. Lancet. 1988;2:12771280 .

20. Larkai EN, Smith JL, Lidsky MD, Graham DY. Gastroduodenal mucosa and dyspeptic symptoms in arthritic patients during chronic nonsteroidal anti-inflammatory drug use. Am J Gastroenterol. 1987;82:1153-1158.

21. Roth SH, Bennett RE. Nonsteroidal anti-inflammatory drug gastropathy. Recognition and response. Arch Intern Med. 1987;147:2093-2100.

22. Wolfe F, Kleinheksel SM, Spitz PW, et al. A multicenter study of hospitalization in rheumatoid arthritis: Frequency, medical-surgical admissions, and charges. Arthritis Rheum. 1986;29:614-619. 